\title{
Mechanisms of microemulsion enhancing the oral bioavailability of puerarin: comparison between oil-in-water and water-in-oil microemulsions using the single-pass intestinal perfusion method and a chylomicron flow blocking approach
}

This article was published in the following Dove Press journal:

International Journal of Nanomedicine

18 November 2013

Number of times this article has been viewed

\author{
Tian-Tian Tang ${ }^{1,2,3}$ \\ Xiong-Bin $\mathrm{Hu}^{1,2,3}$ \\ De-Hua Liao ${ }^{1,2,3}$ \\ Xin-Yi Liu ${ }^{1,2,3}$ \\ Da-Xiong Xiang ${ }^{1,2,3}$ \\ 'Department of Pharmacy, The \\ Second Xiangya Hospital, Central \\ South University, Changsha, People's \\ Republic of China; ${ }^{2}$ Institute of \\ Clinical Pharmacy, The Second Xiangya \\ Hospital, Central South University, \\ Changsha, People's Republic of China; \\ ${ }^{3}$ Key Laboratory for New Technology \\ of Chinese Medicine Preparations of \\ Hunan Province, Changsha, People's \\ Republic of China
}

\begin{abstract}
The purpose of the present work was to determine the mechanisms by which microemulsions (MEs) enhance the oral bioavailability of puerarin. The in situ perfusion method was used in rats to study the absorption mechanisms of an oil-in-water $(\mathrm{O} / \mathrm{W})$ microemulsion $(\mathrm{O} / \mathrm{W}-\mathrm{ME})$ and a water-in-oil (W/O) microemulsion (W/O-ME). The possibility of lymphatic transport of the MEs was investigated using a chylomicron flow blocking approach. The results for the absorption mechanisms in the stomach and intestines indicated that the absorption characteristics of the O/W-ME and W/O-ME depend on the segment. The W/O-ME had higher internal membrane permeability than the O/W-ME. The results of the lymphatic transport analyses showed that both the O/W-ME and W/O-ME underwent lymphatic transport and that this pathway was a major contributor to the oral bioavailability of MEs. Furthermore, the type of ME can significantly affect the absorption of puerarin through the lymphatic system due to the oil content and the form of the microemulsion after oral administration. In conclusion, these data indicate that microemulsions are an effective and promising delivery system to enhance the oral bioavailability of poorly water-soluble drugs.
\end{abstract}

Keywords: microemulsion, lymphatic transport, oral bioavailability, chylomicron

\section{Introduction}

The oral absorption of most currently marketed drugs is limited due to their low solubility in water and low oral bioavailability. To address these problems, many approaches have been developed, including incorporation of the lipophilic component into lipid vehicles such as liposomes and microemulsions (MEs). ${ }^{1}$ The ability of MEs, as promising delivery systems, to enhance the bioavailability of poorly water-soluble drugs has been studied. Currently, there is considerable interest in MEs due to the commercial success of Sandimmune Neoral ${ }^{\circledR}$ (cyclosporine A), Norvir ${ }^{\circledR}$ (ritonavir) and Fortovase ${ }^{\circledR}$ (saquinavir). ${ }^{2}$ However, due to the lack of general formulation guidelines and the limited knowledge about how MEs enhance bioavailability, they are not broadly applied.

MEs are isotropic, thermodynamically stable transparent systems consisting of an oil phase, a water phase, and a surfactant (S), often in combination with a co-surfactant (Co-S). ${ }^{3,4}$ Structurally, MEs are divided into oil-in-water $(\mathrm{O} / \mathrm{W})$, water-in-oil (W/O), and bicontinuous MEs. ${ }^{5}$ In the past few years, many studies have been conducted to demonstrate the enhanced bioavailability of drugs when using MEs. ${ }^{6-8}$ For example,
Correspondence: Da-Xiong Xiang The Second Xiangya Hospital of Central South University, No I 39 Middle Renmin Road, Changsha, Hunan 4I00II, People's Republic of China

Tel +86 73। 85292093

Fax +86 73I 82258487

Email xiangdaxiong@।63.com 
the bioavailability of curcumin was significantly increased when administered to rats in an O/W-ME than when delivered as a suspension. ${ }^{9}$ Lyons et al reported that W/O-MEs are a viable delivery system for $\mathrm{N}$-acetylglucosaminyl-N-acetylmuramyl dipeptide (GMDP), enhancing the bioavailability by 10 -fold when administered intraduodenally. ${ }^{10}$ It has also been reported that W/O-MEs can protect salmon calcitonin from proteolysis and acidic degradation, thus enhancing its absorption in the gastrointestinal tract. ${ }^{11}$ Therefore, MEs have been suggested to positively influence drug absorption in a number of ways, such as protecting the incorporated drugs from oxidation and enzymatic degradation ${ }^{12}$ and enhancing membrane permeability and lymphatic transport. ${ }^{2}$

Lymphatic transport is thought to make a significant contribution to absorption from the intestine as it circumvents the hepatic first-pass effect. ${ }^{13,14}$ Recently, Holm et al showed that halofantrine is also transported by the lymphatic system in dogs when administered in long-chain triglyceride-based self-ME drug delivery systems (SMEDDS) formulations. ${ }^{15}$ There are numerous other examples in the literature demonstrating the enhanced absorption of drugs through the lymphatic system when using MEs. ${ }^{16,17}$ Various animal models are available for the investigation of intestinal lymphatic drug transport. This analysis involves the collection of lymph directly through a catheter and thus requires a high level of surgical skill and has a very low success rate. ${ }^{18}$ Consequently, a chylomicron flow blocking approach developed by Dahan and Hoffman ${ }^{19}$ has been used for lymphatic transport studies. A good correlation between the effects of polysorbate 60 and 80 on the intestinal lymphatic transport of halofantrine in the two models was found. ${ }^{18}$

However, there is no document on the mechanisms of MEs enhancing the oral bioavailability of poorly watersoluble drugs up to date. Therefore, puerarin was used as a model drug to evaluate the feasibility of oral absorption enhancement of MEs. Puerarin, an isoflavone glycoside, is extracted from the root of the traditional Chinese medicines Pueraria lobata (Willd), Ohwi, and Pueraria thomsonni Benth. ${ }^{20}$ Its comprehensive biographical actions have been well-documented by numerous studies, regarding the therapeutic effects on coronary heart disease, ${ }^{21}$ cardiac infarction, ${ }^{22}$ angina pectoris, hypertension, ${ }^{23}$ hypercholesterolemia, ${ }^{24,25}$ hemicranias, as well as diabetic retinopathy. ${ }^{26}$ However, puerarin is a poorly water-soluble drug with low bioavailability upon oral administration. ${ }^{27-29}$ In a previous study, our group prepared a puerarin-loaded O/W-ME and W/O-ME. The oral administration of the drug to rats in MEs significantly enhanced the bioavailability of puerarin, which was consistent with the results reported by $\mathrm{Wu}$ et al. ${ }^{30}$ Although the results of our previous work were encouraging, it is still not known how MEs enhance the bioavailability of puerarin or whether the ME type influences the lymphatic transport of puerarin. To investigate the potential of MEs to serve as effective oral delivery systems for poorly water-soluble drugs, we used the in situ perfusion method to compare the absorption rate and permeability at different sites (stomach, duodenum, jejunum, ileum, and colon) in rats. In addition, the chylomicron flow blocking approach was used to investigate the difference in lymphatic transport between $\mathrm{O} / \mathrm{W}-\mathrm{MEs}$ and W/O-MEs.

\section{Material and methods Reagents and chemicals}

Puerarin was purchased from Nanjing Zelang Medical Technology Co., Ltd. (Nanjing, People's Republic of China). Ethyl oleate, isopropyl myristate, ethyl acetate, ethanol, and propylene glycol were purchased from Sinopharm Group Chemical Reagent Co., Ltd. (Shanghai, People's Republic of China). Polyoxyl 40 hydrogenated castor oil (Cremophor RH40) was donated by BASF SE (Ludwigshafen, Germany). Lecithin with a phosphatidylcholine (PC) content of (70\%-97\%) was obtained from Shanghai Tai-wei Pharmaceutical Co., Ltd. (Shanghai, People's Republic of China). Cycloheximide was purchased from Sigma-Aldrich Chemical Co (St Louis, MO, USA). Acetonitrile and methanol were high performance liquid chromatography (HPLC) grade and were supplied by Tedia Company, Inc (Fairfield, OH, USA). All other chemicals used in the study were of analytical reagent grade.

\section{Animals}

Male Sprague Dawley rats (225-275 g, Central Animal Laboratory of the Second Xiangya Hospital of Central South University, Hunan, People's Republic of China) were used to perform the lymphatic transport study. They were acclimatized for at least 5 days prior to use. All the animal experimental procedures were approved by the institutional animal ethical committee and were in compliance with the National Institutes of Health Guide for Care and Use of Laboratory Animals.

\section{Preparation of a puerarin-loaded O/W-ME and W/O-ME}

The compositions of the formulations were based on those used in our previous study with some modifications. ${ }^{31}$ A pseudoternary phase diagram was constructed to form the ME. The existence of an ME region within the diagrams was observed visually. Variable proportions of oil, S, and 
Co-S were mixed in a glass vial at ambient temperature, and then, the mixture was blended until the oil and the $\mathrm{S}$ / Co-S were completely mixed. Distilled water was added to the mixture, which was then stirred until a transparent ME without drug was obtained. Then, puerarin was added to the mixture, which was stirred to ensure homogeneity. Excess puerarin was removed by centrifugation at 3,000 rpm for 10 minutes. The concentration of puerarin in each formulation was determined before administration by HPLC as described by Liu and Xiang. ${ }^{31}$

\section{Physicochemical characterization of the O/W-ME and W/O-ME}

The average particle size and polydispersity index (PDI) of the resultant $\mathrm{O} / \mathrm{W}-\mathrm{ME}$ and $\mathrm{W} / \mathrm{O}-\mathrm{ME}$ were determined by dynamic light scattering (DLS) using a Zetasizer Nano ZS90 instrument (Malvern Instruments Ltd, Malvern, UK). The morphologies of the MEs were determined using a transmission electron microscope (TEM; JEM-1230; JEOL, Tokyo, Japan). The MEs were diluted 1:50 and mixed by gentle shaking. One drop of each diluted sample was deposited on a film-coated copper grid and allowed to dry before transmission electron microscopy analysis.

\section{Stomach absorption studies}

In situ stomach absorption studies were performed according to the procedure described in the literature. ${ }^{32,33}$ Six Sprague Dawley rats in each group were fasted overnight with free access to water before surgery. The rats were anesthetized using $10 \%$ chloral hydrate $(4.0 \mathrm{~mL} / \mathrm{kg})$ administered intraperitoneally and then restrained in a supine position under an infrared lamp to maintain the body temperature at $37^{\circ} \mathrm{C}$. Thereafter, the abdomen was opened through a longitudinal incision in the midline over a distance of approximately $3 \mathrm{~cm}$. The pylorus of the stomach was cannulated using a polyethylene tube ( $2 \mathrm{~mm}$, inner diameter) ligated with a silk suture. A small incision was made in the cardia, and the stomach was rinsed with simulated gastric fluid using an injection syringe. Puerarin-loaded formulations (O/W-ME, W/O-ME, and suspension) were dissolved or dispersed in simulated gastric fluid at a concentration of $100 \mu \mathrm{g} / \mathrm{mL}$, and $4 \mathrm{~mL}$ of the appropriate formulation was injected into the stomach of each individual rat. The stomach was put back into the abdomen for 2 hours after the pylorus had been ligated. The entire surgical area was covered with a piece of sterilized absorbent gauze wetted with normal saline. At the end of the experiment, the perfusate in the stomach and the simulated gastric fluid that was used to wash the stomach were collected. Then, the obtained solution was diluted to $10 \mathrm{~mL}$, and $200 \mu \mathrm{L}$ of this diluted solution was added to two volumes of absolute methanol. After being vortexed for 2 minutes, the mixture was centrifuged at 13,000 rpm for 5 minutes. Thereafter, a $10 \mu \mathrm{L}$ aliquot of the resulting solution was injected into an HPLC system. The percent absorption of puerarin in the stomach was calculated.

\section{Single-pass intestinal perfusion studies}

The surgical procedure for the single-pass perfusion studies was based on established methods as described previously. ${ }^{33,34}$ Briefly, eighteen male Sprague Dawley rats were divided randomly into three groups of six rats each. The rats were fasted overnight prior to the experiments with free access to water. Anesthesia was induced by the intraperitoneal administration of $10 \%$ chloral hydrate $(4.0 \mathrm{~mL} / \mathrm{kg})$. The pre-disposal treatment of rats was the same as above described. After a $10 \mathrm{~cm}$ long midline incision was made, the duodenum $(1 \mathrm{~cm}$ distal to the pyloric sphincter), jejunum $(15 \mathrm{~cm}$ to the pyloric sphincter), ileum (20 cm proximal to the cecum), and colon ( $2 \mathrm{~cm}$ distal to cecum) of each rat were simultaneously cannulated with polyethylene tubes and ligated at both ends. Care was taken to perform the surgery gently to minimize the damage and maintain intact blood circulation. After being rinsed with physiological saline solution at $37^{\circ} \mathrm{C}$, the selected segments were attached to the perfusion assembly, which included a peristaltic pump (BT100-2F; Baoding Longer Precision Pump Co, Ltd, Hebei, People's Republic of China). The entire surgical area was covered with a piece of sterilized absorbent gauze wetted with normal saline solution at $37^{\circ} \mathrm{C}$. The perfusion solution was obtained by dispersing the MEs in Krebs-Rings perfusate solution at a drug concentration of $100 \mu \mathrm{g} / \mathrm{mL}$ and was incubated in a $37^{\circ} \mathrm{C}$ water bath to maintain the temperature. Before the experiment, the intestinal segments were perfused with the test solution at a flow rate of $0.25 \mathrm{~mL} /$ minute for 30 minutes to achieve absorption equilibrium and stable outflow rates. Subsequently, intestinal perfusate samples were collected over 15 minute intervals for a duration of 1 hour $(15,30,45$, 60 minutes) in $5 \mathrm{~mL}$ glass vials. At the end of the experiment, all samples, including the perfusion samples from both the inlet and outlet drug solutions at different time points, were immediately assayed by HPLC. The samples were prepared for HPLC analysis by adding $200 \mu \mathrm{L}$ of perfusion sample which was added to two volumes of absolute methanol, and after vortexing for 2 minutes, the mixture was centrifuged at $13,000 \mathrm{rpm}$ for 5 minutes. A $10-\mu \mathrm{L}$ aliquot of the supernatant was injected into the HPLC system. All vials were weighed before and after perfusion. The perfused intestinal segments 
between two cannulas were excised without dragging, and their lengths were measured using silk thread. The absorption rate constant $\left(K_{a}\right)$ and apparent permeability coefficients $\left(P_{a p p}\right)$ were calculated using the following equations:

$$
\begin{gathered}
V_{\text {in }}=m_{\text {in }} / \rho_{\text {in }} \\
V_{\text {out }}=m_{\text {out }} / \rho_{\text {out }} \\
K_{a}=\left(1-\frac{C_{\text {out }} V_{\text {out }}}{C_{\text {in }} V_{\text {in }}}\right) \frac{Q}{V} \\
P_{\text {app }}=\frac{-Q \ln \left(\frac{C_{\text {out }}}{C_{\text {in }}}\right)\left(\frac{V_{\text {out }}}{V_{\text {in }}}\right)}{2 \prod r l}
\end{gathered}
$$

where $m_{\text {in }}$ is the weight of the inlet solution, $m_{\text {out }}$ is the weight of the exiting solution, $\rho_{i n}$ is the density of the inlet solution, $\rho_{\text {out }}$ is the density of the exiting solution, $V_{\text {in }}$ is the volume of the inlet solution, $V_{\text {out }}$ is the volume of the exiting solution, $Q$ is the flow rate ( $\mathrm{mL} / \mathrm{minute}$ ) of the inlet solution, $C_{i n}$ is the concentration $(\mu \mathrm{g} / \mathrm{mL})$ of the drug in the inlet solution, $C_{\text {out }}$ is the concentration $(\mu \mathrm{g} / \mathrm{mL})$ of the drug in the exiting solution, $V$ is the volume of the perfused segment $(\mathrm{mL}), l$ is the length of the intestinal segment $(\mathrm{cm})$, and $r$ is the radius of the intestinal segment $(\mathrm{cm})$.

\section{Lymphatic transport studies}

Before the experiment, 36 rats were fasted overnight (12-18 hours) with free access to water, and they were randomly divided into six groups. The rats were treated with either an intraperitoneal injection of $3 \mathrm{mg} / \mathrm{kg}$ cycloheximide in saline $(0.6 \mathrm{mg} / \mathrm{mL})$ or with an equal volume of saline 1 hour before the experiment. ${ }^{18}$ The six groups of rats received the puerarin-loaded O/W-ME, W/O-ME or suspension with a dose of $100 \mathrm{mg} / \mathrm{kg}$ by gavage. The suspension was prepared by mixing puerarin with water and was diluted to a definite volume. Blood $(0.5 \mathrm{~mL})$ was collected from the retro-orbital plexus in heparinized tubes at $0,0.08,0.17,0.33,0.5,0.75$, $1,1.5,2,4,6,8$, and 12 hours after drug administration.

The plasma was separated immediately by centrifugation for 10 minutes at $13,000 \mathrm{rpm}$ and then stored at $-20^{\circ} \mathrm{C}$ until analysis. Blood samples were analyzed by HPLC. Plasma $(100 \mu \mathrm{L})$ was added to a $1.5 \mathrm{~mL}$ screw-capped tube along with $300 \mu \mathrm{L}$ of the internal standard (tectoridin) solution (40 $\mu \mathrm{g} / \mathrm{L})$. After being vortexed for 2 minutes, the mixture was centrifuged at 13,000 rpm for 10 minutes. Then, $200 \mu \mathrm{L}$ of the organic layer was removed, evaporated and then reconstituted in $50 \mu \mathrm{L}$ of methanol. Next, this re-dissolved solution was centrifuged at 13,000 rpm for 10 minutes, and
$20 \mu \mathrm{L}$ of supernatant was used for HPLC analysis. The stationary phase, an Agilent $\mathrm{C}_{8}$ column $(4.6 \mathrm{~mm} \times 250 \mathrm{~mm}$, 5 Mm; Agilent Technologies, Santa Clara, CA, USA), was kept at $30^{\circ} \mathrm{C}$. The mobile phase used for the gradient elution consisted of acetonitrile (A) and water (B). The elution program was as follows: from 0 to 8 minutes, $\mathrm{A}: 11 \%$ to $22 \%$, B: $89 \%$ to $78 \%$; and from 8 to 15 minutes, $\mathrm{A}: 22 \%$ to $11 \% \mathrm{~B}: 78 \%$ to $89 \%$. The flow rate was $1.0 \mathrm{~mL} / \mathrm{minute}$, and a wavelength of $250 \mathrm{~nm}$ was used for detection.

There was no significant interference resulting from the blank plasma under these conditions. For this analysis method, the limit of detection was $50 \mathrm{ng} / \mathrm{mL}$, and the linear range was $0.05-25.0 \mu \mathrm{g} / \mathrm{mL}$. The mean absolute recoveries of puerarin and the internal standard from rat plasma were $94.6 \% \pm 3.45 \%$ and $93.4 \% \pm 3.04 \%$, respectively $(n=5)$. Puerarin and the internal standard were stable in rat plasma after storage for 1 month at $-20^{\circ} \mathrm{C}$ and freeze-thawing three times.

\section{Statistical analysis}

The results are reported as the mean \pm standard deviation (SD). Statistical analysis was performed by one-way analysis of variance. The Student-Newman-Keuls multiple comparison test was used to analyze the potential differences between the formulations. The results were considered significant if $P<0.05$.

\section{Results and discussion Pseudo ternary phase diagram study}

The constructed pseudo ternary phase diagrams are provided in Figure 1. A clear and transparent ME was identified in the ME zone. The optimal ME was selected from this area. As shown in Table 1, O/W-ME and W/O-ME were composed of $2.1 \%$ ethyl oleate, $18.9 \%$ Cremophor RH40/propylene glycol, $79.0 \%$ water and $35.7 \%$ ethyl oleate, $53.6 \%$ lecithin/ethanol and $10.7 \%$ water, respectively.

\section{Characterization of the O/W-ME and W/O-ME}

The particle size and size distribution have substantial effects on the stability of an ME system. The average droplet sizes of the MEs measured by DLS are shown in Table 1, and these sizes were consistent with the transmission electron microscopy results (Figure 2). The size distribution is represented by the PDI, which takes a value between 0 and 1 , with lower values indicating better distributions. In the present work, the PDIs of both the O/W-ME and W/O-ME were always lower than 0.300 , which might be due to the high-pressure homogenization process. As shown in Table 1, the solubility 
A

Water

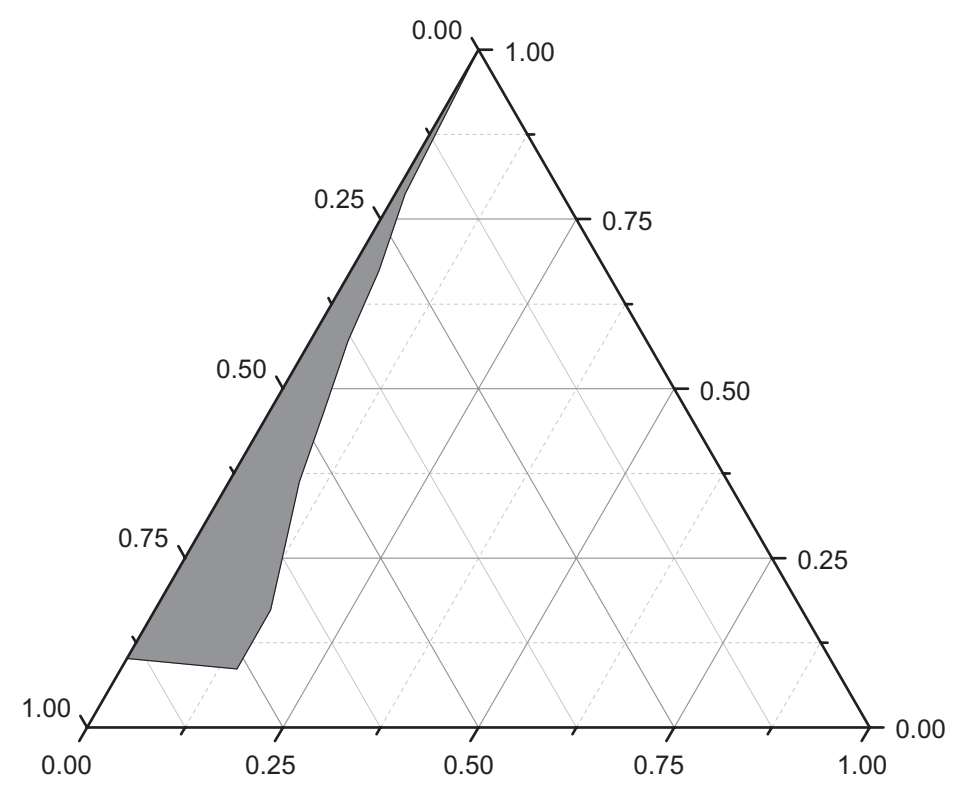

S/Co-S $(2: 1)$

Oil

B

Oil

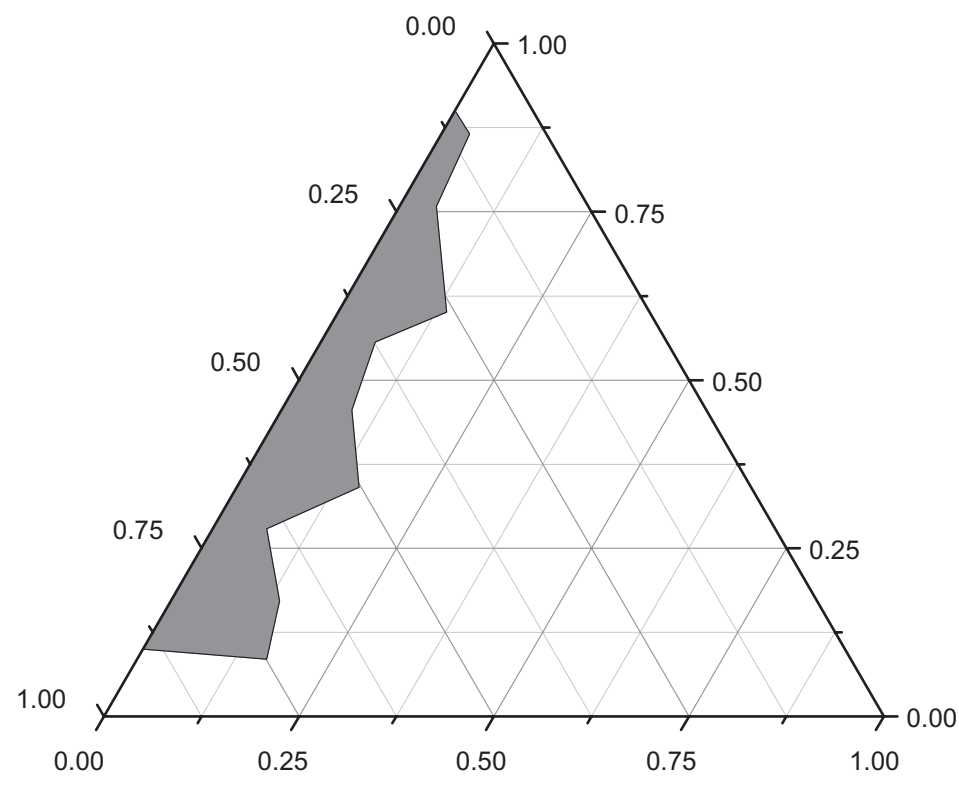

S/Co-S (1:1)

Water

Figure I Pseudo ternary phase diagrams composed of (A) O/W-ME: Cremophor RH40 and propylene glycol as surfactant and co-surfactant (2:I, w/w), (B) W/O-ME: lecithin and ethanol as surfactant and co-surfactant $(\mathrm{I}: \mathrm{I}, \mathrm{w} / \mathrm{w})$, while ethyl oleate was used as the oil phase. The grey area represents the ME existence range.

Abbreviations: Co-S, co-surfactant; ME, microemulsion; O, oil; S, surfactant; W, water; w, weight.

of puerarin in the MEs $(11.32 \pm 0.16$ and $23.14 \pm 0.32 \mathrm{mg} /$ $\mathrm{mL}$ in the O/W-ME and W/O-ME, respectively) was dramatically improved compared with that in aqueous solution $(4.58 \mathrm{mg} / \mathrm{mL}){ }^{35}$ The different Ss used in the formulations could be responsible for the differences in solubility, as Cre- mophor RH40 was used in the O/W-ME, whereas lecithin was used in the W/O-ME. The solubility of puerarin is higher in lecithin than in Cremophor RH40, which may explain why the solubility of puerarin in the W/O-ME was greater than that in the O/W-ME. 
Table I Formulations and droplet sizes of microemulsions and puerarin content

\begin{tabular}{|c|c|c|c|c|c|c|c|}
\hline \multicolumn{4}{|c|}{ Formulations } & \multirow{2}{*}{$\begin{array}{l}\text { Composition } \\
\text { (\%, w/w) } \\
\text { Oil:S:Co-S:W } \\
\end{array}$} & \multicolumn{2}{|l|}{ Droplet size } & \multirow{2}{*}{$\begin{array}{l}\text { Puerarin content } \\
(\mathrm{mg} / \mathrm{mL})\end{array}$} \\
\hline $\mathbf{R x}$ & Oil & $\mathbf{S}$ & Co-S & & Mean $(\mathrm{nm})$ & PDI & \\
\hline O/W-ME & Ethyl oleate & Cremophor RH40 & Propylene glycol & $2.1: 12.6: 6.3: 79.0$ & $13.50 \pm 0.58$ & $0.098 \pm 0.008$ & $11.32 \pm 0.16$ \\
\hline W/O-ME & Ethyl oleate & Lecithin & Ethanol & $35.7: 26.9: 26.7: 10.7$ & $24.04 \pm 1.02$ & $0.102 \pm 0.013$ & $23.14 \pm 0.32$ \\
\hline
\end{tabular}

Note: Each value is the mean \pm standard deviation of three separate determinations.

Abbreviations: Co-S, co-surfactant; ME, microemulsion; O, oil; PDI, polydispersity index; S, surfactant; W, water; w, weight; Rx, prescription.

\section{Stomach absorption studies}

There is limited absorption of oral drugs in the stomach; however, in the present study, the absorption percentages of the suspension, $\mathrm{O} / \mathrm{W}-\mathrm{ME}$ and $\mathrm{W} / \mathrm{O}-\mathrm{ME}$ in the stomach were $16.25 \% \pm 5.10 \%, 20.16 \% \pm 7.00 \%$ and $25.66 \% \pm 10.10 \%$, respectively $(\mathrm{n}=6)$. Although no significant difference was found between the O/W-ME and W/O-
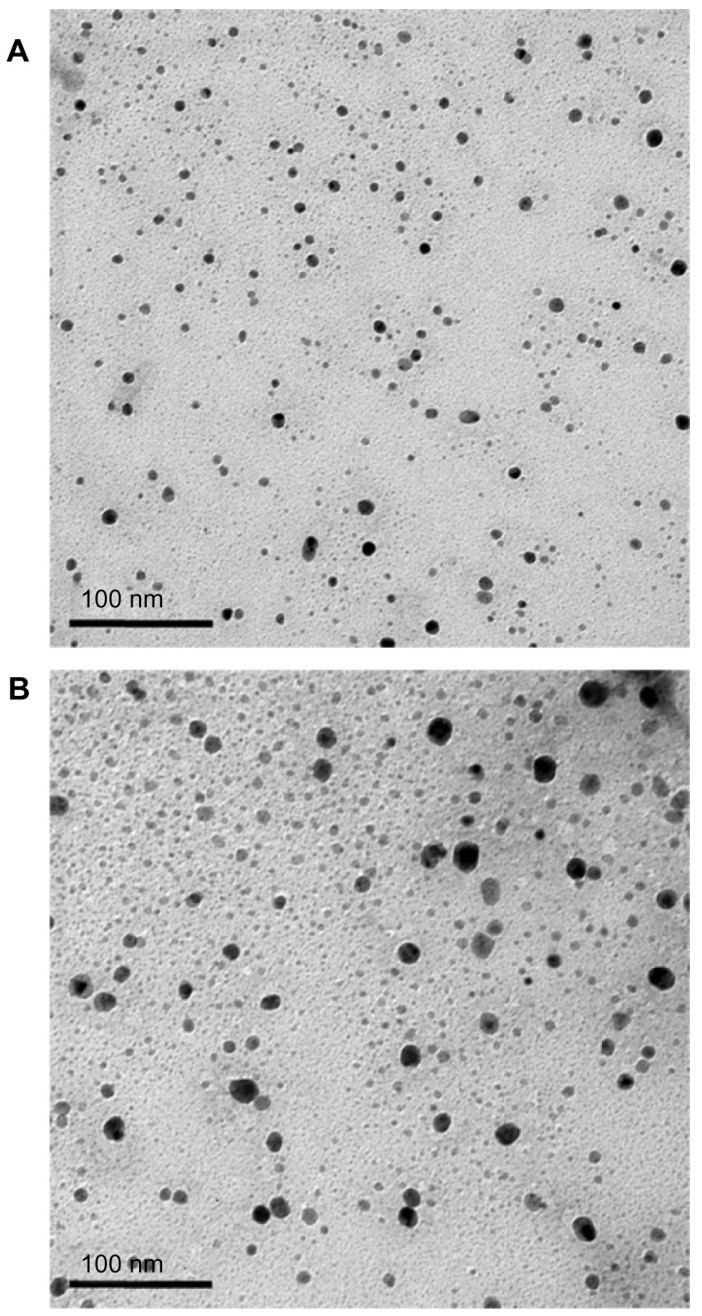

Figure 2 Transmission electron microscopy analysis of microemulsions: (A) O/W-ME and (B) W/O-ME.

Note: Scale bar: $100 \mathrm{~nm}$

Abbreviations: $M E$, microemulsion; $\mathrm{O}$, oil; $\mathrm{W}$, water.
ME, there was a definite trend toward a higher absorption percentage when compared with the suspension. This trend indicated that, to some extent, puerarin absorption can be improved by the use of MEs. This enhanced absorption might be the result of the MEs increasing the compatibility between the drug and the mucous membrane of the stomach. In a previous study, MEs were demonstrated to improve the absorption of drugs in the stomach, with the ME droplets subsequently dispersing in the stomach, allowing them to be readily absorbed ${ }^{29}$ Thus, we assumed that the first absorption site of puerarin-loaded MEs is the stomach.

\section{Single-pass intestinal perfusion studies}

The detection limit of puerarin was $1.875 \mu \mathrm{g} / \mathrm{mL}$, and the method was demonstrated to be linear over the concentration range of $3.75-60.0 \mu \mathrm{g} / \mathrm{mL}$. The mean recovery of puerarin after intestinal perfusion was $97.67 \% \pm 0.85 \%(n=5)$.

In the intestinal absorption studies, the absorption characteristics of the O/W-ME, W/O-ME and suspension were assessed in four different intestinal segments. The absorption rate constant $\left(K_{a}\right)$ and apparent permeability coefficients $\left(P_{a p p}\right)$ obtained using in situ perfusion in the single-pass intestinal perfusion (SPIP) model for the four different intestinal segments are presented in Figures 3 and 4, respectively. The results indicate that puerarin MEs could be absorbed in all intestinal segments. In addition, there were significant differences in absorption between the MEs and the suspension.

Comparisons of the $K_{a}$ and $P_{a p p}$ for these formulations obtained by in situ perfusion in the SPIP model are presented in Figures 5 and 6. The data show that the W/O-ME had higher $K_{a}$ and $P_{a p p}$ values than the other two formulations $(P<0.05)$ in all four intestinal segments. These results indicate that the absorption of the W/O-ME in all intestinal segments was significantly better than the absorption of the two other formulations.

For the O/W-ME formulation, the ileum and colon were the primary sites of absorption, which is consistent with the 


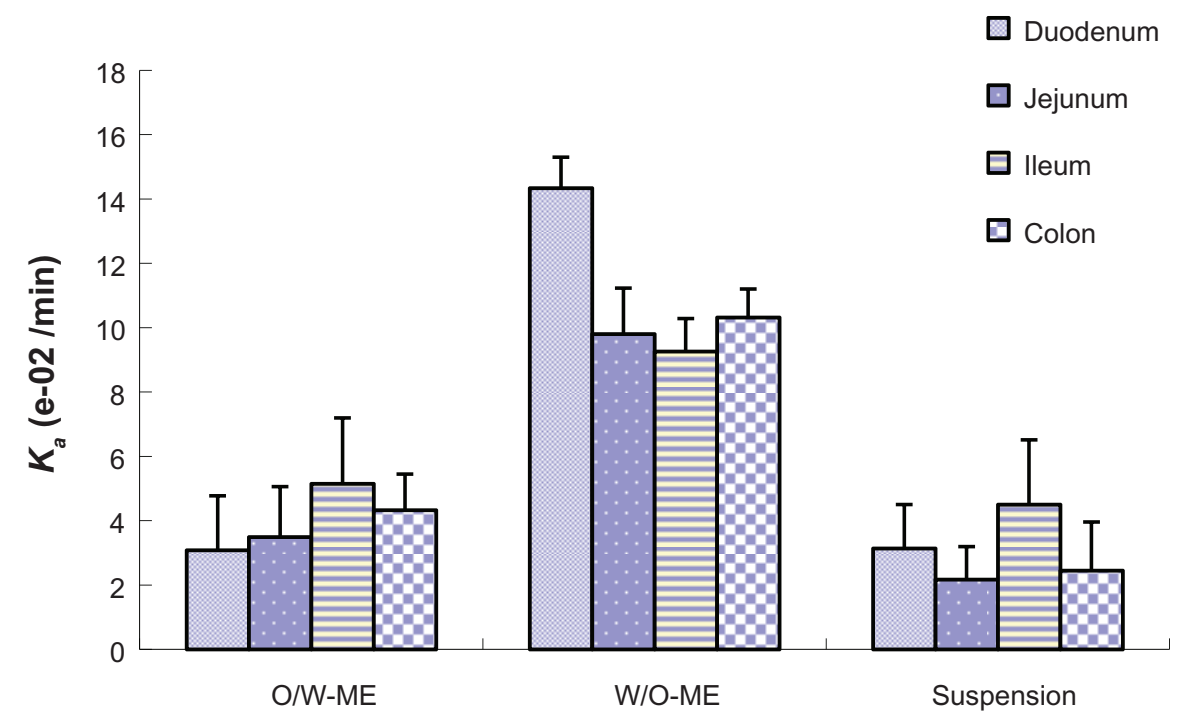

Figure 3 The $K_{a}$ obtained for in situ perfusion in the SPIP model for four different intestinal segments. The data are presented as the mean \pm SD ( $n=6$ ).

Abbreviations: $K_{a}$, absorption rate constant; ME, microemulsion; min, minute; O, oil; SD, standard deviation; SPIP, single-pass intestinal perfusion; W, water.

previous conclusion that there are more Peyer's patches and M-cells in the ileum and colon than in other segments. ${ }^{36}$ However, MEs may enter the bloodstream via the uptake of M-cells, which needs to be confirmed by further research though a few studies have mentioned it. ${ }^{16,23}$ As presented in Figures 3 and 4, the W/O-ME had higher absorption levels in all four intestinal segments than the other formulations. The main intestinal segments in which the W/O-ME was absorbed were the duodenum and colon. In addition, and most likely to a small extent, the W/O-ME could be absorbed as the result of the temporary opening of the epithelial tight junctions much more than the O/W-ME. These reasons might be able to explain why the W/O-ME showed a better absorption than the $\mathrm{O} / \mathrm{W}-\mathrm{ME}$.

\section{Lymphatic transport of the O/W-ME and W/O-ME}

Intestinal lymphatic drug transport has been increasingly emphasized in recent years. To quantify the role that the lymphatic route plays, a chylomicron flow blocking approach, as described by Dahan and Hoffman, ${ }^{19}$ was used to estimate the contribution of the lymphatic transport of the MEs. This approach was used to block chylomicron secretion from the basolateral side of the enterocytes, and reliable results have been achieved. A

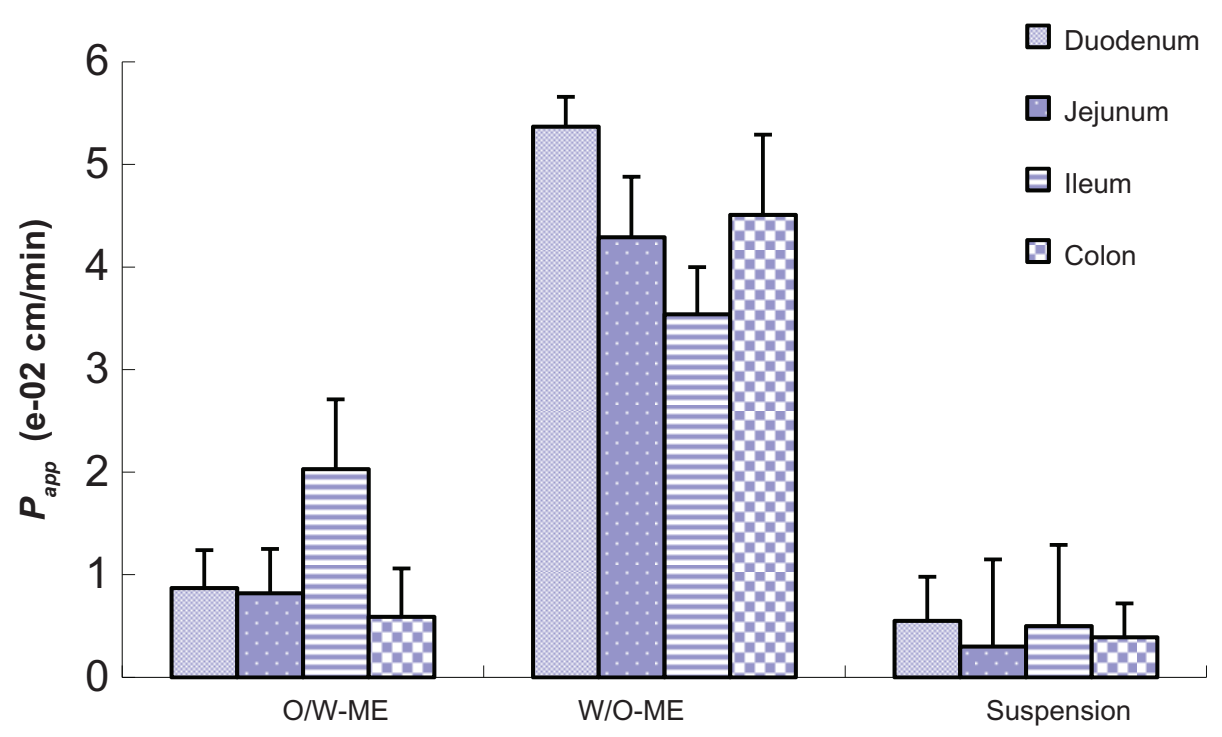

Figure 4 The $P_{a p p}$ obtained for in situ perfusion in the SPIP model for four different intestinal segments. The data are presented as the mean \pm SD ( $n=6$ ). Abbreviations: $P_{a p p}$, apparent permeability coefficients; ME, microemulsion; min, minute; $O$, oil; SD, standard deviation; SPIP, single-pass intestinal perfusion; W, water. 


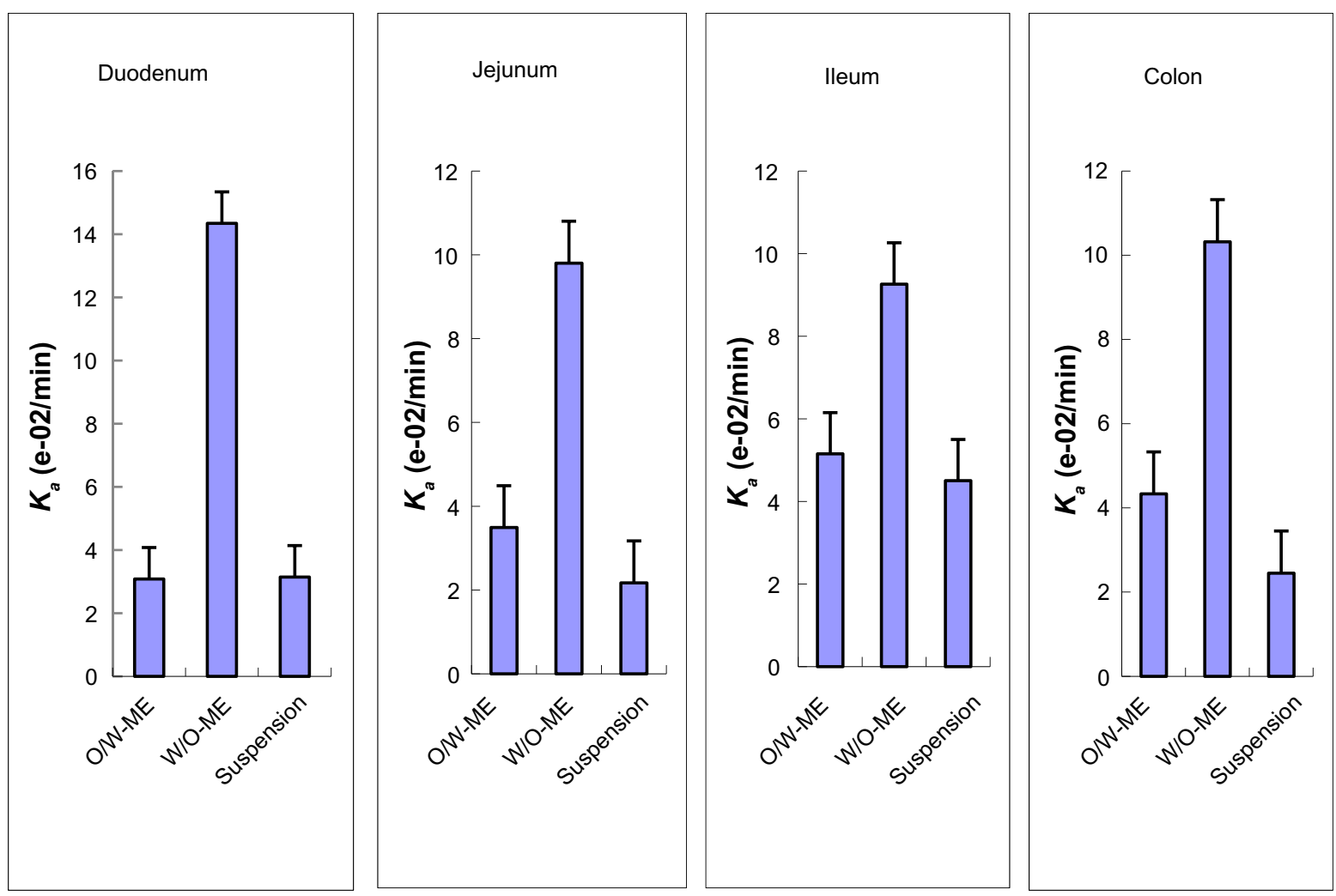

Figure 5 The $K_{a}$ obtained for in situ perfusion in the SPIP model for the suspension, O/W-ME and W/O-ME. The data are presented as the mean \pm SD ( $n=6$ ). Abbreviations: $K_{a}$, absorption rate constant; ME, microemulsion; min, minute; $\mathrm{O}$, oil; SD, standard deviation; SPIP, single-pass intestinal perfusion; W, water.
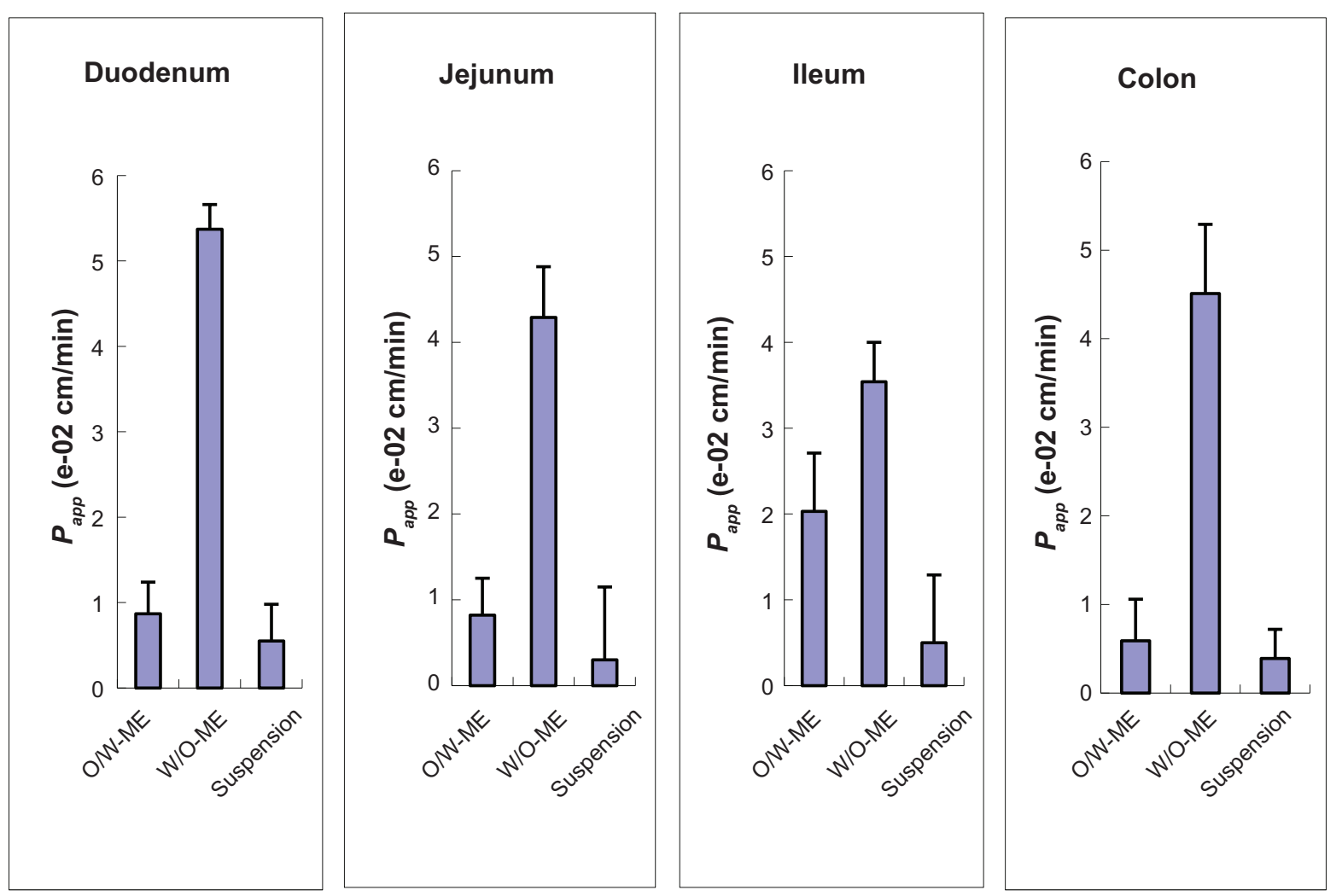

Figure 6 The $P$ obtained for in situ perfusion in the SPIP model for the suspension, O/W-ME and W/O-ME. The data are presented as the mean \pm SD ( $n=6$ ). Abbreviations: $P_{a p p}$, apparent permeability coefficients; ME, microemulsion; min, minute; $O$, oil; SD, standard deviation; SPIP, single-pass intestinal perfusion; W, water. 
good correlation was found between the lymphatic transport of vitamin $\mathrm{D}$ in the conscious, mesenteric lymph-cannulated rat model and in the chylomicron flow blocking model using cycloheximide. ${ }^{19}$ It has been reported that the fraction transported by the lymphatic pathway can be determined by subtracting the fraction transported to the systemic circulation in the rats pretreated with cycloheximide from the total bioavailability in animals pretreated with saline. ${ }^{16,18}$

The puerarin plasma concentration-time profiles obtained after the oral administration of the O/W-ME, W/O-ME, and

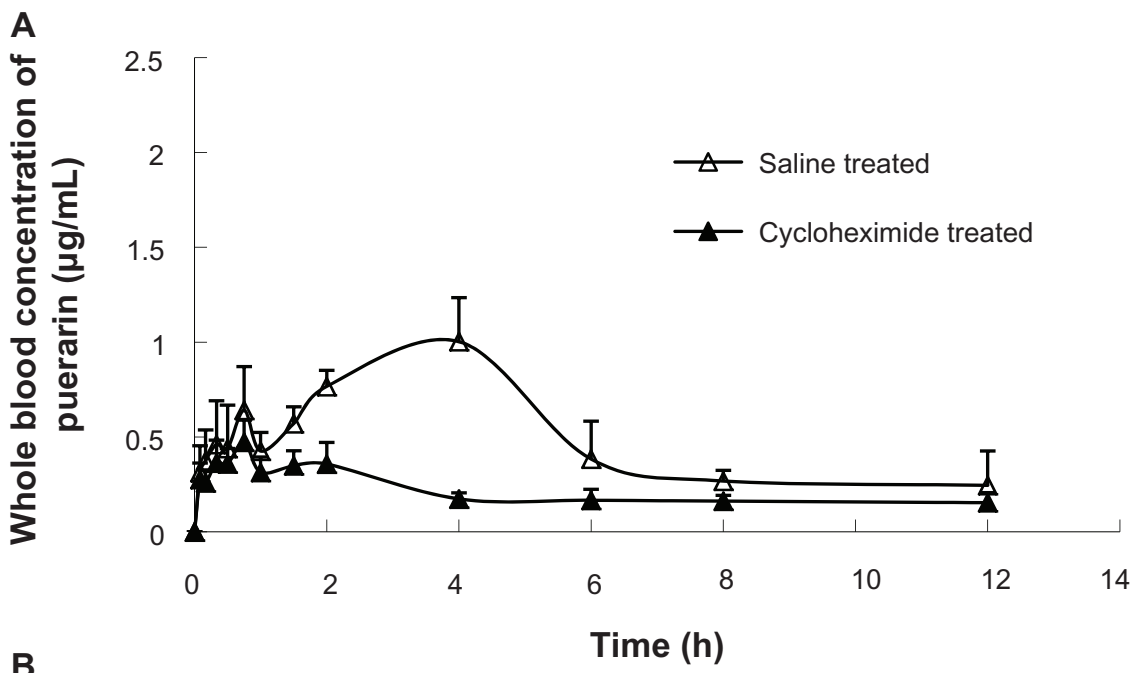

B
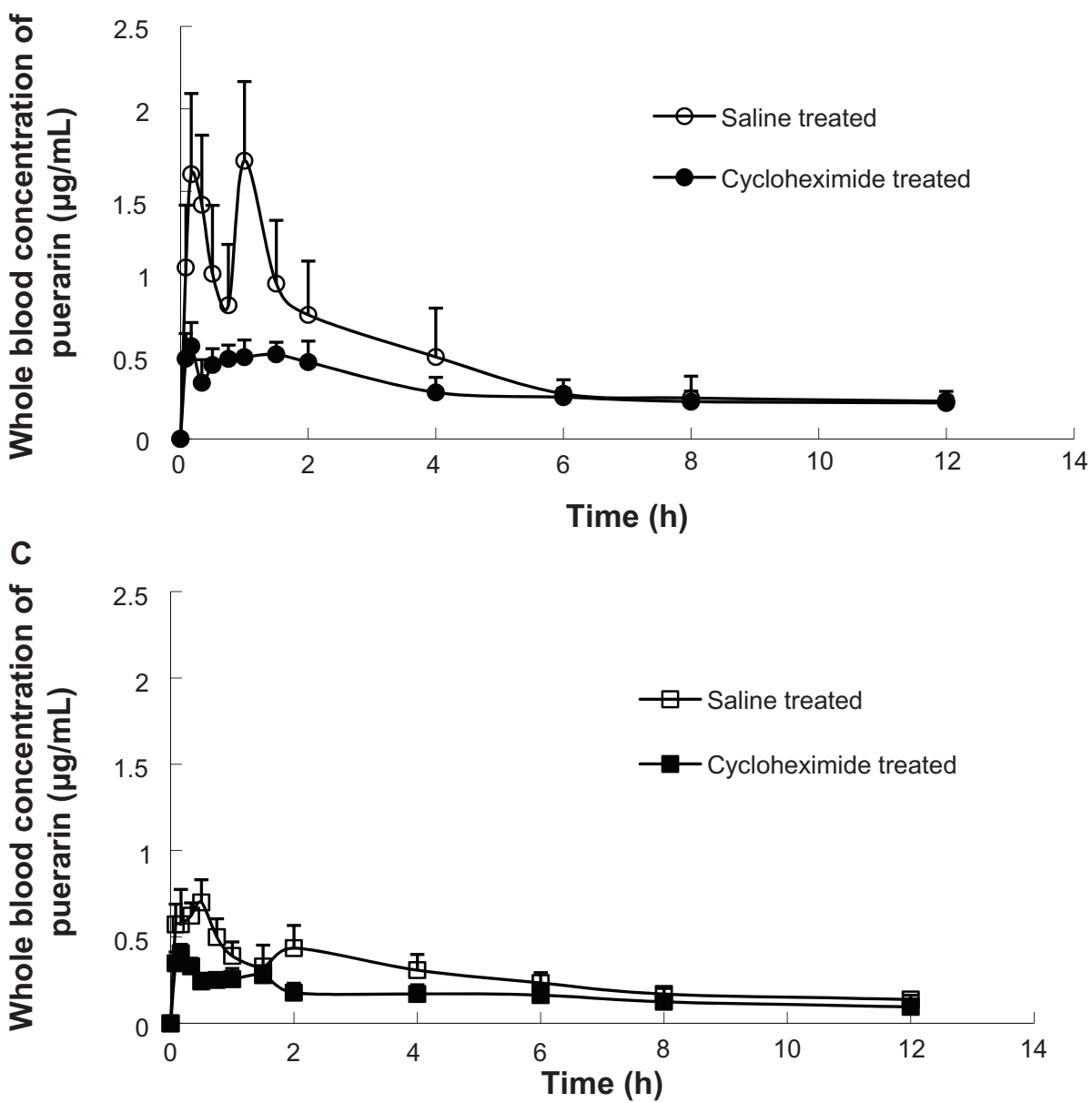

Figure $\mathbf{7}$ The whole blood concentration versus time profiles of puerarin after intragastric administration in the form of (A) an O/W-ME, (B) a W/O-ME, and (C) a suspension at a dose of $100 \mathrm{mg} / \mathrm{kg}$ to cycloheximide- and saline-treated rats $(\mathrm{n}=6)$.

Abbreviations: h, hours; ME, microemulsion; O, oil; W, water. 
Table 2 Pharmacokinetic parameters obtained following the oral administration of puerarin microemulsions and a puerarin suspension in a single dose of $100 \mathrm{mg} / \mathrm{kg}$ to cycloheximide- and saline-treated rats ( $\mathrm{n}=6$, mean \pm standard deviation)

\begin{tabular}{|c|c|c|c|c|c|c|}
\hline \multirow{2}{*}{$\begin{array}{l}\text { Pharmacokinetic } \\
\text { parameters }\end{array}$} & \multicolumn{3}{|c|}{ Cycloheximide treated rats } & \multicolumn{3}{|c|}{ Saline treated rats } \\
\hline & O/W-ME & W/O-ME & Suspension & O/W-ME & W/O-ME & Suspension \\
\hline $\mathrm{AUC}(\mu \mathrm{g} \cdot \mathrm{h} / \mathrm{mL})$ & $2.4 I 5 \pm 0.279 *$ & $3.600 \pm 0.425^{*}$ & $1.837 \pm 0.317^{*}$ & $5.335 \pm 1.646^{\#}$ & $5.636 \pm 1.358^{\#}$ & $2.895 \pm 0.527$ \\
\hline $\mathrm{C}_{\max }(\mu g / \mathrm{mL})$ & $0.468 \pm 0.098 *$ & $0.618 \pm 0.102 *$ & $0.408 \pm 0.048$ & $1.029 \pm 0.208$ & $1.966 \pm 0.362$ & $0.740 \pm 0.099$ \\
\hline $\mathrm{T}_{\max }(\mathrm{h})$ & $0.750 \pm 0.158$ & $0.931 \pm 0.731$ & $0.361 \pm 0.323$ & $0.792 \pm 0.436$ & $0.611 \pm 0.430$ & $0.444 \pm 0.086$ \\
\hline
\end{tabular}

Notes: $* P<0.05$ versus saline treated rats as the control; ${ }^{*} P<0.05$ versus suspension in saline treated rats.

Abbreviations: $A \cup C$, area under the curve; $C_{\text {max }}$, maximum concentration; $h$, hour; $M E$, microemulsion; $O$, oil; $T_{\max }$, time of maximum concentration; $W$, water.

suspension to rats pretreated with saline and cycloheximide are presented in Figure 7, and the pharmacokinetic parameters are listed in Table 2. The bioavailability was determined by computing the area under the curve (AUC) of the drug plasma profile. It is clear that the bioavailability of puerarin following oral administration was significantly higher for the O/W-ME and W/O-ME than for the suspension in saline-treated rats $(P<0.05)$. It can be concluded that the MEs allowed the absorption process to proceed for a longer period of time. Significant differences were found between the maximum concentration $\left(\mathrm{C}_{\max }\right)$ values for the $\mathrm{O} / \mathrm{W}-\mathrm{ME}$ and the W/O-ME. However, no differences were observed in the AUC values. Compared with the saline-treated rats, the AUCs of both the O/W-ME and W/O-ME for the rats pretreated with cycloheximide were less than half of that for the control $(P<0.05)$. The $\mathrm{C}_{\max }$ values of the O/W-ME and W/O-ME decreased from $1.029 \pm 0.208$ to $0.468 \pm 0.098$ and from $1.966 \pm 0.362$ to $0.618 \pm 0.102$ $(P<0.05)$, respectively. The results indicate that blocking chylomicron flow had some impact on drug absorption. In addition, these results suggest that MEs can significantly affect the intestinal lymphatic transport of puerarin.

Occasionally, a double-peak phenomenon was observed in the plasma profiles, as shown in Figure 7. This phenomenon may be explained by the presence of two or more absorption sites in the intestines or irregular gastric emptying. ${ }^{37,38}$ Double peaks were also observed in a recent study in which puerarin was administered orally $(10 \mathrm{mg} / \mathrm{kg})$ to dogs from tablets. ${ }^{39}$ It is not possible to definitively determine the mechanisms responsible for the observed double peak phenomena based on the obtained data, but because this behavior was observed for the oral puerarin suspension as well as for the MEs, it seems unlikely that this phenomenon is caused by the MEs.

In the present study, a tendency toward increased AUC and $\mathrm{C}_{\max }$ values was found in both cycloheximide- and salinetreated rats after the oral administration of the W/O-ME compared with the oral administration of O/W-ME $(P<0.05)$. Thus, we postulate that there are two reasons for the greater absorption-enhancing effect of the W/O-ME. First, the W/O$\mathrm{ME}$ could be transformed into an $\mathrm{O} / \mathrm{W}$ or $\mathrm{W} / \mathrm{O} / \mathrm{W}$ emulsion after it is diluted, and both of these types of emulsions can increase cell membrane fluidity, thus improving the permeability of drug molecules.$^{40}$ Cheng et al reported a W/O-ME formulation for the oral delivery of earthworm fibrinolytic enzyme, demonstrating that the $\mathrm{W} / \mathrm{O} / \mathrm{W}$ emulsion might be a primary converted formulation that improves the stability of the earthworm fibrinolytic enzyme in harsh gastric fluid and intestinal juices when the W/O-ME was administered orally. ${ }^{41}$ Second, the high oil content of the W/O-ME $(35.65 \%)$ used in the present study may lead to an increase in the lymphatic transport of the drug relative to that with the $\mathrm{O} / \mathrm{W}-\mathrm{ME}(2.10 \%)$. In other words, the formulation including more content of oil resulted in more lymphatic transport of the drug and enhanced oral bioavailability. Previous data suggest that lipids play an important role in the lymphatic transport of lipid-based formulations. ${ }^{13,17}$

These results might confirm the transport of MEs through the lymph, but the extent of the contribution of M-cells to this lymphatic transport is still unknown. The primary advantage of intestinal lymphatic drug transport is that drugs absorbed via the intestinal lymphatic system are protected from hepatic first-pass metabolism because the mesenteric lymph, in contrast to the portal blood, enters the systemic circulation directly without first passing through the liver. Therefore, intestinal lymphatic transport might have a substantial beneficial impact on drug bioavailability after oral administration, especially for drugs that are deactivated by hepatic metabolism. In addition, the results of the chylomicron flow blocking experiments confirm that both the $\mathrm{O} / \mathrm{W}-\mathrm{ME}$ and W/O-ME were absorbed via the lymphatic route.

\section{Conclusion}

In conclusion, the data obtained in this study highlight the importance of the lymphatic transport of MEs in the enhanced oral bioavailability of puerarin. Single-pass intestinal perfusion studies showed that the first absorption site of 
MEs may be the stomach. In addition, the absorption of the W/O-ME was much better than that of the O/W-ME in all the intestinal segments. Additionally, with the chylomicron flow blocking model, the present study demonstrated that the type of ME can significantly affect the absorption of drugs via the lymphatic pathway. All of these results suggest that the enhanced oral bioavailability of puerarin can be attributed to the use of the MEs. Importantly, MEs may be promising delivery systems to enhance the oral bioavailability of poorly water-soluble drugs. Additional studies are needed to determine the precise mechanism by which MEs enhance the oral bioavailability of drugs.

\section{Acknowledgments}

This work was supported by the National Science Foundation of Hunan Province, People's Republic of China (08JJ3053).

\section{Disclosure}

The authors report no conflicts of interest in this work.

\section{References}

1. Gursoy RN, Benita S. Self-emulsifying drug delivery systems (SEDDS) for improved oral delivery of lipophilic drugs. Biomed Pharmacother. 2004;58(3):173-182.

2. Talegaonkar S, Azeem A, Ahmad FJ, Khar RK, Pathan SA, Khan ZI. Microemulsions: a novel approach to enhanced drug delivery. Recent Pat Drug Deliv Formul. 2008;2(3):238-257.

3. Schulman JH, Stoeckenius W, Prince LM. Mechanism of formation and structure of micro emulsions by electron microscopy. JPhys Chem. 1959;63(10):1677-1680.

4. Lawrence MJ, Rees GD. Microemulsion-based media as novel drug delivery systems. Adv Drug Deliv Rev. 2000;45(1):89-121.

5. Lu Y, Qi J, Wu W. Absorption, disposition and pharmacokinetics of nanoemulsions. Curr Drug Metab. 2012;13(4):396-417.

6. Wu W, Wang Y, Que L. Enhanced bioavailability of silymarin by self-microemulsifying drug delivery system. Eur J Pharm Biopharm. 2006;63(3):288-294.

7. He CX, He ZG, Gao JQ. Microemulsions as drug delivery systems to improve the solubility and the bioavailability of poorly water-soluble drugs. Expert Opin Drug Deliv. 2010;7(4):445-460.

8. Zeng Z, Zhou G, Wang X, et al. Preparation, characterization and relative bioavailability of oral elemene o/w microemulsion. Int $J$ Nanomedicine. 2010;5:567-572.

9. Cui J, Yu B, Zhao Y, et al. Enhancement of oral absorption of curcumin by self-microemulsifying drug delivery systems. Int J Pharm. 2009;371(1-2):148-155.

10. Lyons KC, Charman WN, Miller R, Porter CJ. Factors limiting the oral bioavailability of $\mathrm{N}$-acetylglucosaminyl- $\mathrm{N}$-acetylmuramyl dipeptide (GMDP) and enhancement of absorption in rats by delivery in a waterin-oil microemulsion. Int J Pharm. 2000;199(1):17-28.

11. Fan Y, Li X, ZhouY, et al. Improved intestinal delivery of salmon calcitonin by water-in-oil microemulsions. Int J Pharm. 2011;416(1): 323-330.

12. Sarciaux J, Acar L, Sado P. Using microemulsion formulations for oral drug delivery of therapeutic peptides. Int J Pharm. 1995;120(2): 127-136.

13. Caliph SM, Charman WN, Porter CJ. Effect of short-, medium-, and long-chain fatty acid-based vehicles on the absolute oral bioavailability and intestinal lymphatic transport of halofantrine and assessment of mass balance in lymph-cannulated and non-cannulated rats. $J$ Pharm Sci. 2000;89(8):1073-1084.
14. Sanjula B, Shah FM, Javed A, Alka A. Effect of poloxamer 188 on lymphatic uptake of carvedilol-loaded solid lipid nanoparticles for bioavailability enhancement. J Drug Target. 2009;17(3): 249-256.

15. HolmR, Porter CJ,Edwards GA, MüllertzA, Kristensen HG, Charman WN. Examination of oral absorption and lymphatic transport of halofantrine in a triple-cannulated canine model after administration in self-microemulsifying drug delivery systems (SMEDDS) containing structured triglycerides. Eur J Pharm Sci. 2003;20(1):91-97.

16. Sun $M, Z$ Zhai $X, X u e K$, et al. Intestinal absorption and intestinal lymphatic transport of sirolimus from self-microemulsifying drug delivery systems assessed using the single-pass intestinal perfusion (SPIP) technique and a chylomicron flow blocking approach: linear correlation with oral bioavailabilities in rats. Eur J Pharm Sci. 2011;43(3):132-140.

17. Porter CJ, Charman WN. Intestinal lymphatic drug transport: an update. Adv Drug Deliv Rev. 2001;50(1-2):61-80.

18. Lind ML, Jacobsen J, Holm R, Müllertz A. Intestinal lymphatic transport of halofantrine in rats assessed using a chylomicron flow blocking approach: the influence of polysorbate 60 and 80. Eur J Pharm Sci. 2008;35(3):211-218.

19. Dahan A, Hoffman A. Evaluation of a chylomicron flow blocking approach to investigate the intestinal lymphatic transport of lipophilic drugs. Eur J Pharm Sci. 2005;24(4):381-388.

20. Wong KH, Li GQ, Li KM, Razmovski-Naumovski V, Chan K. Kudzu root: traditional uses and potential medicinal benefits in diabetes and cardiovascular diseases. $J$ Ethnopharmacol. 2011;134(3): 584-607.

21. Wu L, Qiao H, LiY, Li L. Protective roles of puerarin and Danshensu on acute ischemic myocardial injury in rats. Phytomedicine. 2007;14(10): 652-658.

22. Zhang $\mathrm{S}$, Chen $\mathrm{S}$, Shen $\mathrm{Y}$, et al. Puerarin induces angiogenesis in myocardium of rat with myocardial infarction. Biol Pharm Bull. 2006;29(5): 945-950.

23. Song XP, Chen PP, Chai XS. [Effects of puerarin on blood pressure and plasma renin activity in spontaneously hypertensive rats.] Zhongguo Yao Li Xue Bao. 1988;9(1):55-58. Chinese.

24. Yan LP, Chan SW, Chan AS, Chen SL, Ma XJ, Xu HX. Puerarin decreases serum total cholesterol and enhances thoracic aorta endothelial nitric oxide synthase expression in diet-induced hypercholesterolemic rats. Life Sci. 2006;79(4):324-330.

25. Chung MJ, Sung NJ, Park CS, et al. Antioxidative and hypocholesterolemic activities of water-soluble puerarin glycosides in HepG2 cells and in C57 BL/6J mice. Eur J Pharmacol. 2008;578(2-3):159-170.

26. Teng Y, Cui H, Yang M, et al. Protective effect of puerarin on diabetic retinopathy in rats. Mol Biol Rep. 2009;36(5):1129-1133.

27. Wu H, Zhou A, Lu C, Wang L. Examination of lymphatic transport of puerarin in unconscious lymph duct-cannulated rats after administration in microemulsion drug delivery systems. Eur J Pharm Sci. 2011;42(4):348-353.

28. Zhang Y, Wang R, Wu J, Shen Q. Characterization and evaluation of self-microemulsifying sustained-release pellet formulation of puerarin for oral delivery. Int J Pharm. 2012;427(2):337-344.

29. Cui S, Zhao C, Chen D, He Z. Self-microemulsifying drug delivery systems (SMEDDS) for improving in vitro dissolution and oral absorption of Pueraria lobata isoflavone. Drug Dev Ind Pharm. 2005;31(4-5): 349-356.

30. Wu H, Lu C, Zhou A, Min Z, Zhang Y. Enhanced oral bioavailability of puerarin using microemulsion vehicle. Drug Dev Ind Pharm. 2009;35(2):138-144.

31. Liu X, Xiang DX. Preparation and evaluation of puerarin microemulsion for oral delivery. Central South Pharmacy. 2007;5(05):451-455.

32. Li H, Zhao X, Ma Y, Zhai G, Li L, Lou H. Enhancement of gastrointestinal absorption of quercetin by solid lipid nanoparticles. J Control Release. 2009;133(3):238-244.

33. Peng Q, Zhang ZR, Sun X, Zuo J, Zhao D, Gong T. Mechanisms of phospholipid complex loaded nanoparticles enhancing the oral bioavailability. Mol Pharm. 2010;7(2):565-575. 
34. Li M, Si L, Pan H, et al. Excipients enhance intestinal absorption of ganciclovir by P-gp inhibition: assessed in vitro by everted gut sac and in situ by improved intestinal perfusion. Int J Pharm. 2011;403(1-2): $37-45$.

35. Yu A, Wang H, Wang J, et al. Formulation optimization and bioavailability after oral and nasal administration in rabbits of puerarinloaded microemulsion. J Pharm Sci. 2011;100(3):933-941.

36. Sato S, Kaneto S, Shibata N, et al. Transcription factor Spi-B-dependent and -independent pathways for the development of Peyer's patch M cells. Mucosal Immunol. 2013;6(4):838-846.

37. Langguth $P$, Lee KM, Spahn-Langguth H, Amidon GL. Variable gastric emptying and discontinuities in drug absorption profiles: dependence of rates and extent of cimetidine absorption on motility phase and $\mathrm{pH}$. Biopharm Drug Dispos. 1994;15(9):719-746.
38. Metsugi Y, Miyaji Y, Ogawara K, Higaki K, Kimura T. Appearance of double peaks in plasma concentration-time profile after oral administration depends on gastric emptying profile and weight function. Pharm Res. 2008;25(4):886-895.

39. Lai J, Liao ZG, Liang XL, Zhao GW, Zhang P, Zhang XH. Study on Pharmacokinetics and Bioequivalence of Multi-Absorption Enhancer and Puerarin Tablet. Chinese Journal of Experimental Traditional Medical Formulae. 2010;16(7):142-147.

40. Qi J, Zhuang J, Wu W, et al. Enhanced effect and mechanism of waterin-oil microemulsion as an oral delivery system of hydroxysafflor yellow A. Int J Nanomedicine. 2011;6:985-991.

41. Cheng MB, Wang JC, Li YH, et al. Characterization of water-in-oil microemulsion for oral delivery of earthworm fibrinolytic enzyme. J Control Release. 2008;129(1):41-48.

\section{Publish your work in this journal}

The International Journal of Nanomedicine is an international, peerreviewed journal focusing on the application of nanotechnology in diagnostics, therapeutics, and drug delivery systems throughout the biomedical field. This journal is indexed on PubMed Central, MedLine, CAS, SciSearch ${ }^{\circledR}$, Current Contents ${ }^{\circledR} /$ Clinical Medicine,
Journal Citation Reports/Science Edition, EMBase, Scopus and the Elsevier Bibliographic databases. The manuscript management system is completely online and includes a very quick and fair peer-review system, which is all easy to use. Visit http://www.dovepress.com/ testimonials.php to read real quotes from published authors. 